\title{
Pattern Recognition from Segmented Images in Automated Inspection Systems
}

\author{
Mira Park ${ }^{1}$, Jesse S. Jin ${ }^{1}$, Sherlock L. Au ${ }^{2}$, Suhuai Luo ${ }^{1}$ \\ 1. The school of Design, Communication \& IT, The University of Newcastle, Australia \\ 2. Multi Base Ltd, Hong Kong \\ mira.park@newcastle.edu.au
}

\begin{abstract}
We present the segmentation of the foreground objects and the identification of the individual objects in the cigarette tin package, so the information will be used for the classification of the acceptable cases or defective cases. Visual inspection and classification of cigarette tin package are very important in manufacturing cigarette products that require high quality package. For the accurate automated inspection and classification, computer vision has been deployed widely in manufacturing. This paper concerned with the problem of identifying the individual cigarette in the tin packing using the image processing and Morphology operations. The identified objects can be used for developing a defect finding system in the cigarette packing industries. The approach has two steps: (i) Colour-based segmentation of the Region of Interests, (ii) identifying of individual object. The segmentation performance was evaluated on 18 images including the good cases and the defective cases.
\end{abstract}

\section{Introduction}

The package inspection systems are continuously conveyed in the manufacturing process. The systems are capable of measuring predetermined parameters of packages, comparing the measured parameters with predetermined values, evaluating from the measured parameters the integrity of the packages and determining whether such packages are acceptable or, alternatively, should be rejected.

Humans are able to find such defects with prior knowledge. Human judgment is influence by expectations and prior knowledge. However, it is tedious, laborious, costly and inherently unreliable due to this subjective nature. Therefore, these traditional visual quality inspection performed by human inspectors has the potential to be replaced by computer vision systems for many tasks. These increased demands for objectivity, consistency and efficiency have necessitated the introduction of the accurate automated inspection systems. The automated inspection systems employing image processing techniques has been developed rapidly, which can quantitatively characterize complex size, shape, colour and texture properties of products.

The accurate automated inspection and classification can reduce human workloads and labour costs while increasing the throughput. In fact, much research have been carried out on automated inspection of tile surface [1], biscuit bake colour [2], colour of potato chip [3], textile fabrics [4], food product [5, 6], and wood $[7,8]$ while relatively little work has been done in automated defect classification, mainly because of the difficult nature of the problem. Computer vision has been used to objectively measure the colour of different food since they provide some obvious advantages over a conventional colorimeter, namely, the possibility of analysing of each pixel of the entire surface of the food, and quantifying surface characteristics and defects $[9,10]$.

The most difficulty is the segmentation of the background and the foreground and the extraction of the important information in the foreground. The segmentation process partitions the digital image into disjoint regions. Segmentation is an essential step in computer vision and automatic pattern recognition processes based on image analysis of foregrounds as subsequent extracted data are highly dependent on the accuracy of this operation. In general, the automated segmentation is one of the most difficult tasks in the image analysis, because a false segmentation will cause degradation of the measurement process and therefore the interpretation may fail.

Visual inspection and classification of cigarette tin package are very important in the manufacturing of cigarette products that require high quality package. For the accurate automated inspection and 
Table 1. The examples of the good cases and the defective cases

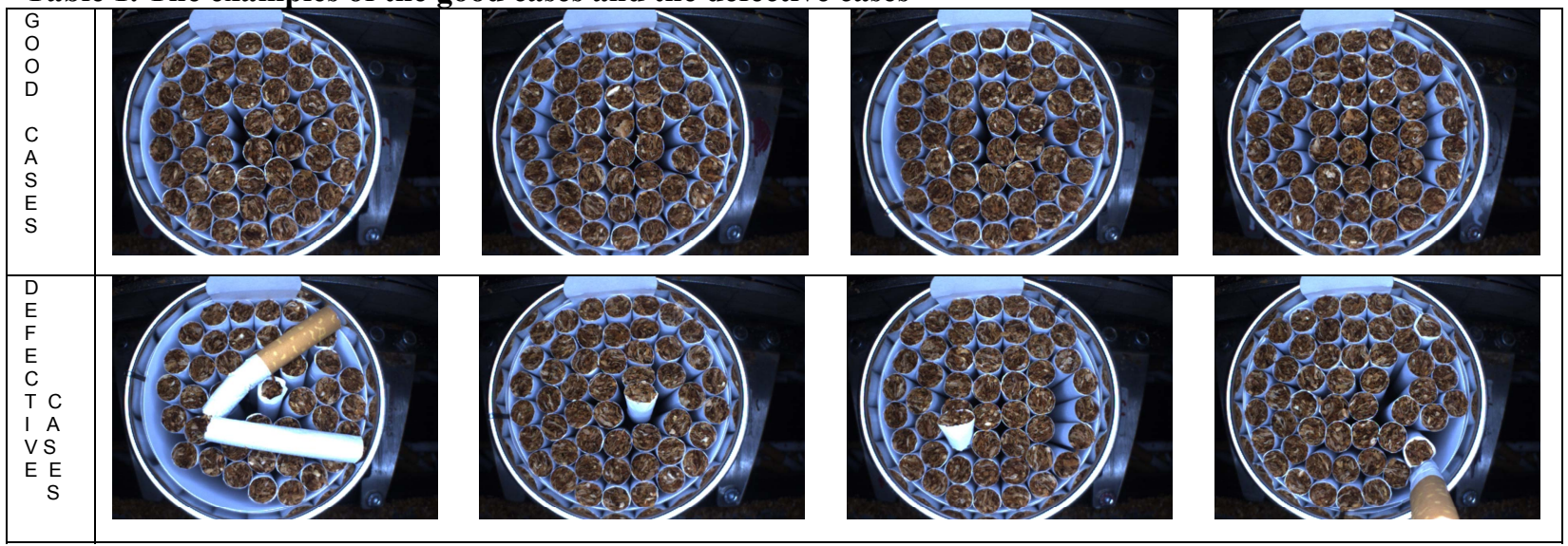

classification, computer vision has been deployed widely in the manufacturing. Computer vision is a novel technology for acquiring and analysing an image of a read scene by computers and other devices in order to obtain information or to control processes. The core technique in computer vision is always related to image processing, which can lead to segmentation, qualification and classification of images and objects of interest within images.

Yeh[2] used Kohonen's selforganising map for identifying baking curves of baked goods, Zhang and Bresee [11] detected defective images in textile fabrics by individually applying simple classification to discriminate knots from slubs according to the ratio of length to width, and Brazakovic [7] employed a pyramid-linking scheme to locate defects in wood and a hierarchical defect classification scheme to classify four types of wood defect.

In this paper, we present the segmentation of the foreground objects and the identification of the individual objects in the cigarette tin package, so the information will be used for the classification of the acceptable cases or defective cases [see Table1].

\section{Methods}

\subsection{Colour-Based Segmentation of the Region of Interests}

We use the $L^{*} a^{*} b^{*}$ colour space to segment colours in an automated fashion. The $L^{*} a^{*} b^{*}$ colour space is a colour-opponent with dimension $L^{*}$ for Lightness and $a^{*}$ and $b^{*}$ for the colour-opponent dimensions, based on nonlinearly-compressed CIE XYZ colour space coordinates $[12,13]$.
Step 1: Convert image from RGB colour space to $\mathrm{L} * \mathrm{a} * \mathrm{~b} *$ colour space.

Colour is a determining factor in the identifying of the objects in an image. With a digital camera it is possible to register the colour of any pixel of the image of the object using three colour sensors per pixel [17]. The most often used colour model is the RGB model in which each sensor captures the intensity of the light in the $\operatorname{red}(\mathrm{R})$, green $(\mathrm{G})$ or blue(B) spectrum, respectively. The human vision system developed a way to correct for the quality of illumination, and to preserve differences in hue. A white paper is perceived as white even in reddish evening light. To allow similar possibilities is colour measurement, CIE (Commission on Internationale de l'Eclairage) defined several more human-related, less illumination-dependent measures of colour, of which the most common is $L^{*} a^{*} b^{*}$ [14].

There are mainly three colours in our input image if we ignore variations in brightness: white, black, brown. The $L^{*} a{ }^{*} b^{*}$ colour space enables to quantify these visual differences since the $L^{*} a^{*} b^{*}$ colour is designed to approximate human vision. It aspires to perceptual uniformity, and its $L^{*}$ component closely matches human perception of lightness. It can thus be used to make accurate colour balance corrections by modifying output curves in the $a^{*}$ and $b^{*}$ components, or to adjust the lightness contrast using the $L^{*}$ component. The $\mathrm{L}^{*} \mathrm{a} * \mathrm{~b} *$ space consists of a luminosity layer $L^{*}$, chromaticity-layer $a^{*}$ indicating where colour falls along the red-green axis, and chromaticity-layer $b^{*}$ indicating where the colour falls along the blueyellow axis. 


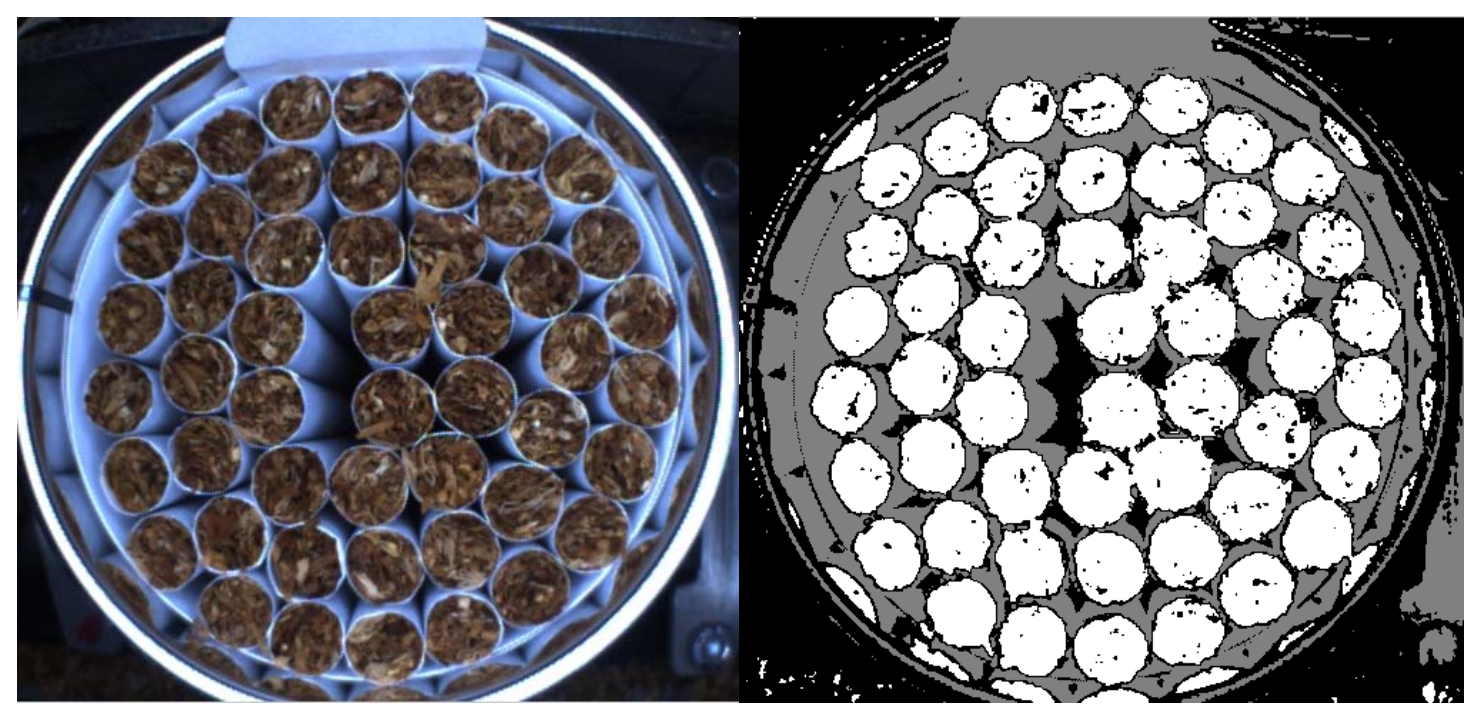

Figure 1. Input image (left) and Image labelled by cluster index (right)

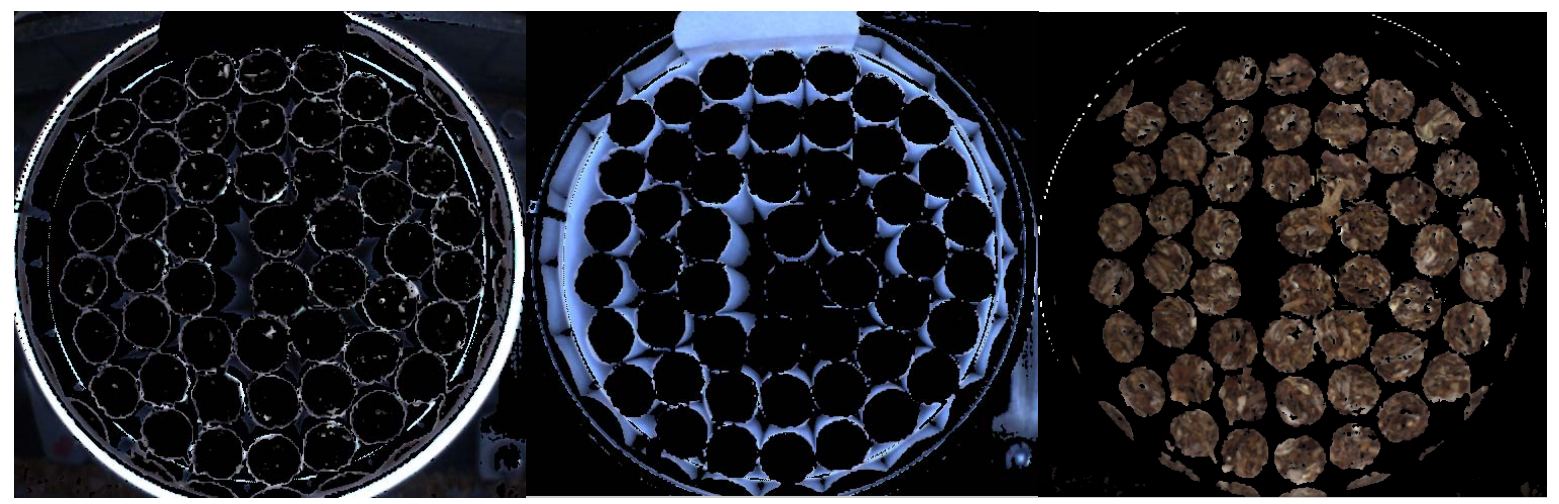

Figure 2. Separated objects by colour. left: objects in cluster 1, middle: objects in cluster 2, right: objects in cluster 3

The methodology used for estimating the RGB $\rightarrow$ $L * a * b *$ transformation consists of two parts [15].

(i) Definition of the model: The model has parameters $\theta_{1}, \theta_{2}, \ldots \theta_{m}$ whose inputs are the RGB variables obtained from the color digital image of a sample, and whose outputs are the $L^{*} a^{*} b^{*}$ variables estimated from the model; and

(ii) Calibration: The parameters $\theta_{1}, \theta_{2}, \ldots, \theta_{m}$ for the model are estimated on the basis of the minimization of the mean absolute error between the estimated variables (model output) $\hat{L^{*}}, a^{*}, b^{*}$ and the $L^{*}, a^{*}, b^{*}$ variables (measured from the sample used in i) through the use of a colorimeter.

\section{Construction of Linear Model}

In this, the simplest model of all, the $\mathrm{RGB} \rightarrow \mathrm{L}^{*} \mathrm{a} * \mathrm{~b}^{*}$ transformation is a linear function of the $(\mathrm{R}, \mathrm{G}, \mathrm{B})$ variables:

$\left[\begin{array}{c}\hat{L^{*}} \\ \hat{a}^{*} \\ \hat{b}^{*}\end{array}\right]=\left[\begin{array}{llll}M_{11} & M_{12} & M_{13} & M_{14} \\ M_{21} & M_{22} & M_{23} & M_{24} \\ M_{31} & M_{32} & M_{33} & M_{34}\end{array}\right]\left[\begin{array}{c}R \\ G \\ B \\ I\end{array}\right]$

The following is an explanation of how the parameters of the first row of matrix $\mathbf{M}$ are obtained; the same explanation is valid for the other rows: We first must define the parameters vector for the model

$$
\theta=\left[\begin{array}{llll}
M_{11} & M_{12} & M_{13} & M_{14}
\end{array}\right]^{T}
$$



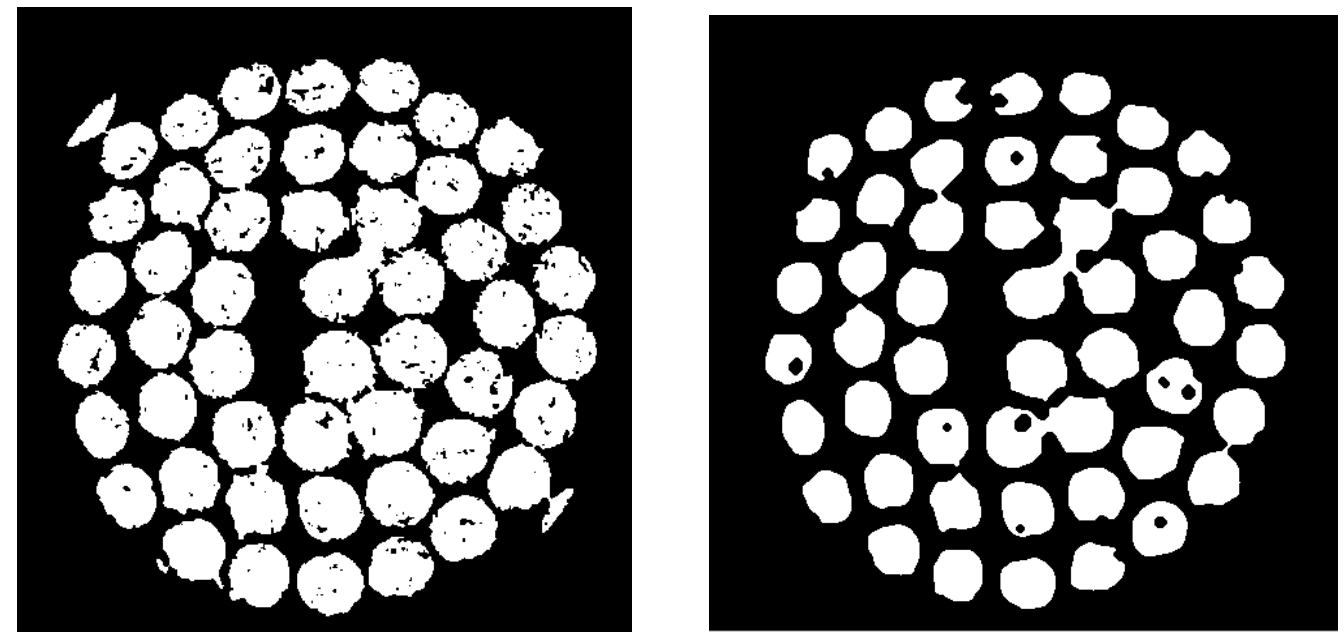

Figure 3. The left image is the binary image of the segmented image and the right image is the simplify image of the left image.

The input matrix with $N$ measurements of $R, G, B$

$X=\left[\begin{array}{cccc}R_{1} & G_{1} & B_{1} & 1 \\ \vdots & \vdots & \vdots & \vdots \\ R_{N} & G_{N} & B_{N} & 1\end{array}\right]$ $L^{*}$

And the output vector with the $N$ measurements of

$y=\left[\begin{array}{lll}L_{1}^{*} & \ldots & L_{N}^{*}\end{array}\right]^{T}$

thus the estimate of $L^{*}$, obtained from the minimization of the norm between measurements and estimated $\|y-\hat{y}\|$, is defined by [16]:

$\hat{y}=X \theta$

where

$$
\theta=\left[X^{T} X\right]^{-1} X^{T} y
$$

The advantage of this model is that it is direct and its solution is not obtained through iterations.

Step2: Classify the colors in ' $\mathrm{a}{ }^{*} \mathrm{~b}^{*}$ ' space using Kmeans clustering

We use K-means clustering to separate groups of objects. K-means clustering treats each object as having a location in space. It finds partitions such that objects within each cluster are as close to each other as possible, and as far from objects in other clusters as possible. K-means clustering requires that you specify the number of clusters to be partitioned and a distance metric to quantify how close two objects are to each other. Since the color information exists in the ' $a * b^{*}$ ' space, the objects are pixels with ' $a$ *' and ' $b$ '' values. K-means clusters the objects into three clusters using the Euclidean distance metric. For every object in the input image, K-means returns an index corresponding to a cluster (see Figure 1). The right most image in Figure 2. is segmented as a Regions of Interest.

\subsection{Identification of individual object}

The identification of objects within an image can be a very difficult task. One way to simplify the problem is to change the greyscale image into a binary image, and morphological operations are used on these binary images. This phrase boundaries individual object (cigarette) and marks the centre of each object for the further process.

Step1: Create a improved binary image by thresholding and morphological operations

First, we produce a binary image from the segmented RGB images. Thresholding is used to convert the segmented image to binary image. The output binary image has values of 1 (white areas in the left image of Figure 3.) for all pixels and 0 (black) for all other pixels. However, the segmentation suffers from inaccuracy because the paper liked colours exist in the cigarette and they are segmented as parts of background. 

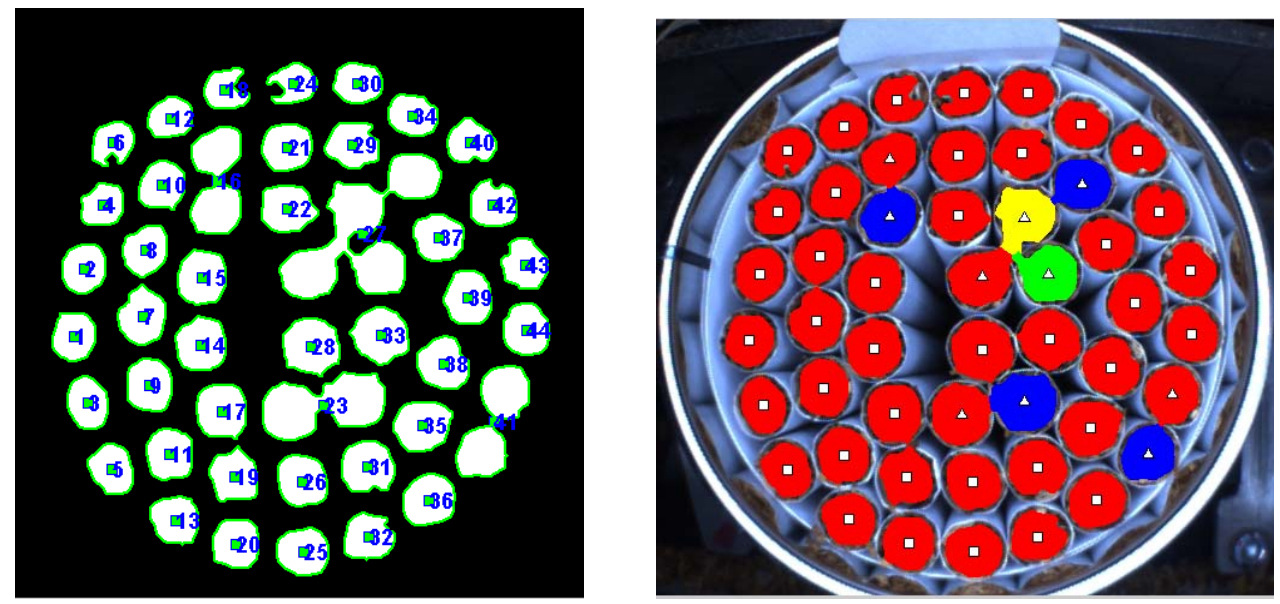

Figure 4. [Left] Labelled Image: green lines are boundary of each object, green squares represent the centre of each object and the objects are labelled by the blue numbers. [Right] Red regions represent the initial segmented regions. Blue, yellow and green regions are newly divided regions from the first segmented region. The white squares represent the centre of the objects and the white triangles represent the new centres of the re-clustered objects.

The other obstacle to identify the individual object is the connection between the objects. These inaccuracies should be reduced before further process. We use morphological operations to reduce the inaccuracies by improving the binary images. Morphological operations are methods for processing binary images based on shapes. As binary images frequently result from segmentation processes on grey level images, the morphological processing of the binary result permits the improvement of the segmentation result. The improved images increase the accuracy. The morphological operation is performed in two steps: (i) The shrink operation removes pixels so that objects without holes shrink to a point, and objects with holes shrink to a connected ring halfway between each hole and the outer boundary. (ii) To fill a small hole, we set a pixel to 1 if five or more pixels in its 3-by-3 neighbourhood are 1's; otherwise, we set the pixel to 0 .

\section{Step2: Label the binary image}

Contiguous regions are labeled to be identified as an individual object. The $k^{\text {th }}$ region includes all elements in the labeled image $\mathrm{L}$ that have value $k$. For example, the pixels labeled 1 make up one object, the pixels labeled 2 make up a second object, and so. Therefore, the number of objects in $\mathrm{L}$ is equal to $\max (\mathrm{L})$.

\section{Step3: Separate the joined objects}

In figure 4 (left), the labeled object 16, 23 and 41 contains two objects and the object 27 contained four objects, therefore, the joined objects should be separated to be an individual object. We use K-mean classification to separate them. The average number of pixels in one cigarette image is 1800 pixels, therefore, to obtain how many individual cigarette in the labeled object, $\mathrm{K}$ is calculated by (Area/unit_pixels) +1 where Area is the area of the labeled object and unit pixels is 1800 . If $\mathrm{K}$ is greater than 1 , the object is re-clustered into K clusters (see Figure 4).

The final image includes only the cigarette parts, and the number of objects in the image and the centre points of each object are obtained from this final image. This information is very useful for the classification of the defective cases.

\section{Experimental Results}

We tested our method based on 8 good cases and 10 defective cases. Our aim is to identify the individual objects, so each object is coloured and the centres are marked. From the good cases, there are $8 * 50 \quad(=400)$ objects and our method segmented correctly all of them $(400$ (true positives) $/(400$ (true positives) +0 (false negatives) $=1$ (sensitivity), specificity $(0$ (true negatives $) /(0$ (true negatives $)+0$ (false positives $)=0$. Therefore, all objects belonging to the tin are classified as foreground. From the defective cases, our method segmented all objects if the size is reasonable (more than 300 pixels), therefore the sensitivity is 1 and there are 6 false positives (0(true negatives) / 0 (true negatives $)+6$ (false negatives $)=0$. We success in our algorithm that can identify almost all individual objects in the tins (see Table 2). 
Table 2 The examples of the good cases and the defective cases

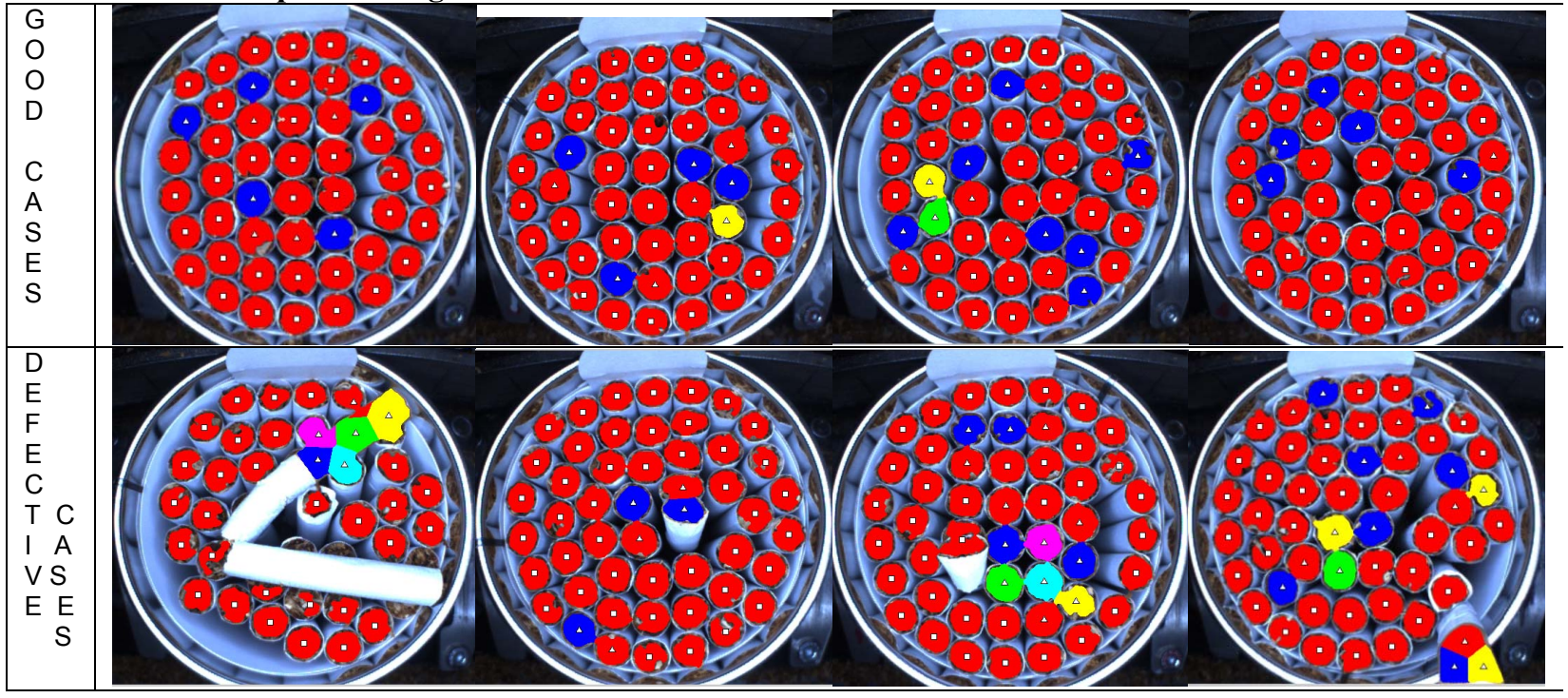

\section{Conclusions}

This paper concerned with the problem of identify of the individual cigarette in the tin packing using the image processing and Morphology operations. The identified objects can be used for developing of defect finding system in the cigarette packing industries. We success in identifying of the individual cigarettes. The approach has two steps: (i) Colour-based segmentation of the Region of Interests, (ii) identifying of individual object. The segmentation performance was evaluated on 18 images including the good cases and the defective cases.

\section{References}

[1] Elbehiery H., Hefnawy A. and Elewa M., Surface Defects Detection for Ceramic Tiles Using Image Processing and Morphological Techniques, Proceedings of World Academy of Science, Engineering and Technology, 5:1307-6884, 2005 [2] Yeh C.H.J., Hamey G.C.L., Westcott T. and Sung K.Y.S, Color Bake Inspection System Using Hybrid Artificial Neural Networks, IEEE International Conference of Neural Networks, 37-42, 1995

[3] Segnini S., Dejmek P. and Öste R., A Low Cost Video Technique for Color Measurement of Measurement of Potato Chips, Lebensm-Wiss. U-Technology, 33:216-222, 1999

[4] Kwak C., Ventura A.J. and Tofand-Sazi K., Automated Defect Inspection and Classification of Leather Fabric, Intelligent Data Analysis, 5:355-370, 2001

[5] Mery D. and Pedreschi F., Segmentation of colour frood imgages using a robust algorithm, Journal of Food Engineering, 66:353-360, 2005
[6] Yam L.K. and Papadakis E.S., A Simple Digital Imaging Method for Measuring and Analyzing Color of Food Surfaces, Journal of Food Engineering, 61:137-142, 2004

[7] Braxakovic D., Beck H. and Sufi N., An approach to defect detection in materials characterized by complex textures, Pattern Recognition 23(1/2): 99- 107, 1990

[8] Kauppinen H., Pautio H. and Silven O., Non-segmenting defect detection and SOM based classification for surface inspection using color vision, Polarization and Color Techniques in Industrial Inspection (SPIE 3826), 270-280, 1999

[9] Brosnan T. and Sun D., Improving quality inspection of food products by computer vision - a review, Journal of Food Engineering 61:3-16, 2004

[10] Du C. and Sun D. Recent Developments in the Applications of Image Processing Techniques for Food Quality Evaluation, Trends in Food Science and Technology, 15:230-249, 2004

[11] Zhang Y.F. and Bresee R.R., Fabric defect detection and classification using image analysis, Textile Research Journal 65(1):1-9, 1995

[12] Hunter, Richard Sewall, Photoelectric Color-Difference Meter. JOSA, Proceedings of the Winter Meeting of the Optical Society of America 38 (7): 661.1948

[13] Hunter, Richard Sewall Accuracy, Precision, and Stability of New Photo-electric Color-Difference Meter. JOSA, Proceedings of the Thirty-Third Annual Meeting of the Optical Society of America 38 (12): 1094.[14] Hunt R.W.G., Measuring Colour, $2^{\text {nd }}$ ed. Ellis Horwood, Ltd, 1991 [15] Leon K., Mery D., Pedreschi F., and Leon J., Color measurement in $L^{*} a^{*} b^{*}$ units from RGB digital images, Food Research International, 39:1084-1091, 2006

[16] Stöderström and Stoica, 1989 T. Stöderström and P. Stoica, System identification, Prentice-Hall, New York 1989 [17] Forsyth D. and Ponce J., Computer vision: a modern approach, Prentice Hall, New Jersey, 2003 